\title{
Robust Wireless Local Area Networks Based on Compressed Sensing
}

\author{
Mohammadreza Balouchestani-Asli \\ Computer, Electrical, and Information Technology Department, Indiana Purdue University Fort Wayne (IPFW), \\ 2101 East Coliseum Boulevard, Fort Wayne, IN 46805-1499, USA; balouchm@ipfw.edu
}

Received: 30 January 2018; Accepted: 12 March 2018; Published: 19 March 2018

\begin{abstract}
A wireless local area network (WLAN) is an important type of wireless network which connotes different wireless nodes in a local area network. Network traffic or data traffic in a WLAN is the amount of network packets moving across a wireless network from each wireless node to another wireless node, which provide the load of sampling in a wireless network. WLAN's network traffic is the main component for network traffic measurement, network traffic control, and simulation. In addition, traffic classification technique is an essential tool for improving the Quality of Service (QoS) in different wireless networks in the complex applications, such as local area networks, wireless local area networks, wireless personal area networks, wireless metropolitan area networks, and wide area networks. Network traffic classification is also an essential component in the products for QoS control in different wireless network systems and applications. Classifying network traffic in a WLAN allows one to see what kinds of traffic we have in each part of the network, organize the various kinds of network traffic in each path into different classes in each path, and generate network traffic matrix in order to identify and organize network traffic, which is an important key for improving the QoS feature. In this paper, a new architecture based on the following algorithms is presented for improving the QoS feature in a wireless local area network: (1) Real-Time Network Traffic Classification (RTNTC) algorithm for WLANs based on Compressed Sensing (CS); (2) Real-Time Network Traffic Monitoring (RTNTM) approach based on CS. This architecture enables continuous data acquisition and compression of WLAN's signals that are suitable for a variety of other wireless networking applications. At the transmitter side of each wireless node, an analog CS framework is applied at the sensing step before an analog to digital converter in order to generate the compressed version of the input signal. At the receiver side of the wireless node, a reconstruction algorithm is applied in order to reconstruct the original signals from the compressed signals with high probability and enough accuracy. The proposed architecture allows reducing Data Delay Probability (DDP) to 15\%, Bit Error Rate (BER) to $14 \%$ at each wireless node, False Detection Rate (FDR) to 25\%, and Packet Delay (PD) to $15 \%$, which are good records for WLANs. The proposed architecture is increased Data Throughput (DT) to $22 \%$ and Signal to Noise (S/N) ratio to $17 \%$, and $10 \%$ accuracy of wireless transmission. The proposed algorithm outperforms existing algorithms by achieving a good level of Quality of Service (QoS), which provides a good background for establishing high quality wireless local area networks.
\end{abstract}

Keywords: real-time network traffic monitoring; compressed sensing; wireless local area networks; bit error rate; sampling rate; signal to noise ratio; real-time network traffic classification; false detection rate; packet delay; quality of service

\section{Introduction}

A wireless computer network can use either infrared or radio frequency technology to transmit and receive information over the air, in order to link a set of computers using a wireless distribution 
method [1-3]. This type of network implements a flexible data communication system frequently augmenting, rather than replacing, a wired LAN within a building or campus. WLANs have become popular for use in many applications, such as the home, school, computer laboratory, industrial automation, and office building [4,5]. One important advantage of WLANs is to simplify networking topologies by enabling multiple computer users or networks to simultaneously share resources, including software and hardware systems in a home or business without additional or intrusive wiring [6,7]. In order to improve the performance of a WLAN, we need to minimize network traffic, load of sampling rate, and power consumption. RTNTM and RTNTC are two important algorithms to improve the QoS for all users. Traditional RTNTM techniques rely on Shannon Nyquist Sampling (SNS) procedure, which suffer from huge load of sampling and power consumption [8]. Classification of network traffic in a WLAN is a strong tool that helps identify different applications and protocols that exist in a wireless network. Various actions, such as monitoring, discovery, control, and optimization, can then be performed on the identified network classes for improving the network performance [8]. Typically, once the packets are classified (identified) as belonging to a particular path or wireless device, different paths of a wireless network, or protocol, they are marked or flagged [8]. These markings or flags help the wireless router to determine appropriate path, routing matrix, routing vector, routing service, routing table, routing approach, and policies to be applied for those flows [8].

A WLAN is a type of different wireless network, such as wireless personal area networks, wireless body area networks, wireless metropolitan area networks, wireless campus area networks, and wireless storage area networks. In the WLANs, the infrared or radio frequency technology can be used to transmit and receive information over the air, in order to link a set of computers using a wireless distribution method [8]. This type of network implements a flexible data communication system frequently augmenting, rather than replacing, a wired LAN within a building or campus. WLANs have become popular for use in many applications, such as the healthcare, business, e-health, e-learning, e-business, home, school, computer laboratory, industrial automation, and office building. One important advantage of WLANs is to simplify networking topologies, such as star and bus, by enabling multiple computer users or networks to simultaneously share resources, including software and hardware systems, and wireless networking devices in a home or business without additional or intrusive wiring. Real-time network traffic classification technique is an essential tool for wireless networks in complex applications, such as wireless local area networks, wireless personal area networks (WPANs), wireless metropolitan area networks (WMANs), wireless campus area networks (WCANs), wireless storage area networks (WSANs), and wide area networks (WANs), in order to improve the quality of the wireless networks and wireless network performance.

In this paper, a new architecture based on the following algorithms is presented for improving the QoS feature in WLANs: (1) RTNTC algorithm for WLANs based on CS; (2) RTNTM approach based on CS. In addition, a new QoS tomography based on RTNTM method is presented that enables us to identify the cause of QoS degradation when such degradation is detected in a WLAN. This architecture enables continuous data acquisition and compression of WLAN's signals that are suitable for a variety of other wireless networking applications. At the transmitter side of each wireless node, an analog CS framework is applied at the sensing step before analog to digital converter, in order to generate the compressed version of the input signal. At the receiver side of wireless node, a reconstruction algorithm is applied in order to reconstruct the original signals from the compressed signals with high probability and enough accuracy. In this work, a new architecture based on the following algorithms is presented for improving the QoS feature in the WLANs: (1) a real-time network monitoring algorithm based on CS theory; (2) a new real-time network classification based on CS theory; (3) a new quantitative quality measure method to identify the cause of QoS degradation. The proposed architecture allows DDP to $15 \%$, BER to $14 \%$ at each wireless node, FDR to $25 \%$, and PD to $15 \%$, which are good records for WLANs. The proposed architecture increased DT to $22 \%$ and S/N ratio to $17 \%$, and $10 \%$ accuracy of wireless transmission. The proposed algorithm outperforms existing algorithms by achieving a good level of QoS, which provides a good background for establishing high quality wireless local area 
networks. This paper is organized as follows. Section 2 reviews related work. Section 3 proposes two new algorithms, RTNTM and RTNTC, based on CS. Section 4 explains the results. Finally, the paper concludes in Section 5 .

\section{Overview of the Related Works}

The conventional sampling approaches have traditionally relied on the SNS procedure. The SNS theory demonstrates that analog and digital signals, images, and videos can be exactly recovered from a set of uniformly spaced samples taken at SNS, which is twice the highest frequency in the signal of interest [9-11]. Therefore, a signal must be sampled at least twice its bandwidth, in order to be represented without error at the receiver side. The traditional approaches based on SNS have two major drawbacks [12-14]. First, they generate huge intolerable samples for many applications with a large bandwidth. Second, even for low signal bandwidths, they produce a large amount of redundant digital samples. That is why it is desirable to reduce the number of acquired samples in a WLAN by utilizing sparsity. The main motivation is to replace the conventional sampling and reconstruction operation with a general random linear measurement process and an optimization scheme in order to recover the original signal from a small number of random measurements in a WLAN [15]. The CS states that if a real-world signal has a sparse representation in a certain transform base, then it is possible to recover the signal with significantly fewer random samples or measurements [16]. CS is a new approach for the acquisition and recovery of sparse signals that enables a sampling rate significantly below the classical Nyquist rate. This theory states a small number of random linear measurements of compressible signals in a WLAN contain enough information to recover and process the original signal at each nose of a WLAN. Sensing and processing of the signals in a WLAN has traditionally relied on the Shannon Sampling theorem. This theorem states that, given a signal of bandwidth $\Omega$, it is sufficient to sample at $2 \Omega$ samples per second to ensure faithful representation and reconstruction. The traditional approach has fallen short lately. First, in many signals, $\Omega$ is so large that the Nyquist rate is unbearable. Second, even for low signal bandwidths, the traditional approach produces a large amount of redundant digital samples, which are costly for wireless communication and severely limit the WLAN's lifetime. Therefore, traditional approaches have two major drawbacks: they generate a huge and intolerable number of samples for applications with large bandwidth and a large amount of redundant digital samples for low bandwidth signals. Three important advantages of a WLAN are (1) the simplicity of its installation; (2) monitoring and management of the network; (3) applying strong security protocols. Installing a wireless LAN system is easy, and can eliminate the need to pull cables through walls and ceilings [17,18]. The physical architecture of WLAN is quite simple. Basic components of a WLAN are access points (APs) and Network Interface Cards (NICs)/client adapters, Switches, Routers, Bridges, and Repeaters $[19,20]$. The use of WLANs is becoming very widespread, and the variety of usage patterns are increasing. Consequently, the factors that affect the end-to-end QoS of WLANs are becoming much more diverse. These factors are not only due to the different applications of WLANs, but also to other attributes of wireless communications, such as the type of access network (e.g., WPANs, WMANs, and WANs [20]. Network traffic or data traffic in a WLAN is the amount of network packets moving across a wireless network from each wireless node to another wireless node, which provides the load of sampling in the network [21,22]. WLAN's network traffic is the main component for network traffic measurement, network traffic control, and simulation $[23,24]$. The new RTNTM is presented in this paper. In addition, in this paper, the quality of QoS is improved based on a new tomography method that enables us to identify the cause of QoS degradation when such degradation is detected in a WLAN. This method involves using a signal processing technique called CS to reconstruct a signal from a series of sampled measurements, assuming that the signal vector is sparse, which means that it consists of a few non-zero elements, and the remaining element's value is zero. The amount of network traffic in WLANs is rapidly increasing, and traffic patterns are changing due to the widespread use of different kinds of applications for WLANs. Therefore, this is a key to understand traffic characteristics and QoS through network monitoring and measurement in order to 
ensure effective network management $[25,26]$. For this purpose, it is very important to identify the cause of QoS degradation, which leads to a challenging networking issue. Related works on improving the QoS include various methods that have been proposed based on the SNS theorem for network monitoring and network measurement purposes. Based on the existing methods, failure localization and network tomography identify the location of a link/node failure or wireless nodes, and estimate the QoS of each link, then, by sending probe wireless packets between multiple pairs of edge nodes, wireless nodes, and segments. These methods are focused on locating link/node failures or estimating link QoS inside a WLAN with the probe packets, rather than analyzing the end-to-end QoS and identifying the QoS degradation factors, including wireless devices and applications by collecting QoS measurement from various wireless networking devices, on which we are focusing. WLAN's network traffic is the main component for network traffic measurement, network traffic control, and simulation. It is very important to classify network traffic. Classifying network traffic in a WLAN allows to see what kinds of traffic we have in each part of the network, organize the various kinds of network traffic in each path into traffic classes in each path, and generate network traffic matrix in order to identify and organize network traffic, which is the foundation for improving the QoS feature. Network traffic classification in WLANs offers an important ability to automatically recognize the application that has generated a stream of packets of a wireless node from the direct and passive observation of the individual packets from other wireless nodes or wireless devices $[27,28]$. This ability is instrumental to a number of active wireless nodes and wireless devices in a WLAN. Classification of packets is an important tool that is required to enable any traffic management operations, from differentiating traffic pricing and treatment to security operations [29]. The information provided by traffic classification in a WLAN is extremely important for improving the quality of the wireless network. Traditional traffic classification techniques rely on Shannon SNS procedure, which suffers from huge load of sampling and power consumption [30].

The existing classification methods are based on two main approaches to classifying network traffic in a WLAN:

- Classifying the packet behavior based on the payload, that is, Payload-Based Classification (PBC). In this method, packets are classified based on the fields of the payload, such as source wireless node, or destination wireless node, or both.

- Classification based on statistical algorithms in a WLAN that use statistical analysis of the network traffic behavior, like inter-packet arrival, session time, frequency, packet delay, bandwidth, accuracy, and so on.

In order to improve the QoS in a WLAN, we need to divide the network traffic into different classes on a wireless network-identifying some important parameters for each packet to which packets belong is crucial when assigning them to the different class.

The fundamental goal of the proposed RTNTC algorithm is to solve difficult wireless network management problems in different wireless network applications. The result of RTNTC algorithm could be applied for Internet service providers (ISPs) and their wireless networking equipment vendors. It is a key tool for network operators to see what is flowing over their wireless networks in order to support their various networking goals and protocols. RTNTC algorithm is a core part of wireless network automated intrusion detection systems to detect patterns indicative of denial of service attacks, trigger automated re-allocation of wireless network resources for priority routing algorithms, or identify different paths for the wireless networks. The conventional classification approaches have traditionally relied on the SNS procedure. The SNS theory demonstrates that analog and digital signals, images, and videos can be exactly recovered from a set of uniformly spaced samples taken at SNS, which is twice the highest frequency in the signal of interest. Therefore, a signal must be sampled at least twice its bandwidth in order to be represented without error on the receiver side. The traditional approaches based on SNS have two major drawbacks. First, they generate huge intolerable samples for many applications with a large bandwidth. Second, even for low signal 
bandwidths, they produce a large amount of redundant digital samples. That is why it is desirable to reduce the number of acquired samples by utilizing sparsity. The main motivation is to replace the conventional sampling and reconstruction operation with a general random linear measurement process and an optimization scheme in order to recover the original signal from a small number of random measurements. Ensemble clustering techniques involve the generation of clusters using more than one process in order to identify an ultimate clustering by consensus. Many of the existing methods are expensive, which restricts their applications for clustering of large and highly dimensional datasets in the WLANs. The main drawbacks for the existing clustering and classification algorithm are (1) they are not able to provide real-time clustering and classification; (2) the classification is performed offline, due to lack performance of clustering method; (3) they are not effective for wireless communication systems because they consume huge power; (4) they suffer from low processing time and speed. Therefore, we need to design a new classification approach based on CS for the different applications, such as WLANs, WPANs, WMANs, and WANs. The CS method involves using a signal processing technique to reconstruct a signal from a series of sampled measurements, assuming that the signal vector is sparse to the small number of non-zero samples.

In addition, classifying network traffic enables us to allocate network resources to deliver optimal performance for different types of wireless networks. RTNTM is a strong tool which we need, not only to fix network problems in time, but also to prevent network failure, to detect inside and outside threats, to improve the quality of our networking, and make good decisions for network planning for different wireless networking applications. As the wireless networking systems and applications keep growing, the need of network monitoring, control, and analysis tools have been increasing. Monitoring wireless network traffic in different applications, such as WLAN, WPAN, WMAN, and WAN, has become increasingly challenging in terms of number of hosts, protocol proliferation, and probe placement topologies. Network monitoring and management in different types of wireless networks, especially in WLANs, have become more and more important in a modern complicated network, including WLAN, WPAN, WMAN, and WAN networks. Based on a new application of wireless networks, there is a critical need to design a new network monitoring tool for both LAN and WAN, in order to maintain the network system stability and availability, such as to fix network problems on time or to avoid network failure, to ensure the network security strength, and to make good decisions for network planning. There are various kinds of tools dealing with the network monitoring and analysis of different types of wireless networks, such as tools used by the Simple Network Management Protocol (SNMP), Monitoring Tools Integrated with NMP, Web Tools, Windows Management Instrumentation (WMI), Sniffing, and Network flow monitoring and analysis.

\section{Proposed Algorithms}

The CS theory says many natural signals in a WLAN are sparse, or near sparse, in the sense that they have concise representations when expressed in the convenient basis. The CS scenario enables continuous waveform sampling data acquisition and compression that are suited for a variety of wireless networking applications [30]. The CS procedure that is presented in this paper addresses both the energy and telemetry bandwidth constraints of WLANs. The CS theory states sparse or compressible signals in a WLAN can be well recovered when minimizing $\ell_{1}$ norm optimization, while satisfying some important conditions for the random measurement matrix $\Phi$ and orthogonal basis $\Psi[31]$.

\subsection{RTNTM Algorithm}

The proposed algorithm is based on a conventional Fast Fourier Transformation (FFT) to check signal sparsity in a WLAN [31]. These signals have $K$ non-zero coefficients and (N-K) zero coefficients with $\mathrm{K} \leq \mathrm{M}$, and can be well recovered using $M$ projections or measurements, such that $\mathrm{K} \leq \mathrm{M}<<\mathrm{N}$. As a result, the number of non-zero coefficients is small; the CS theory can, therefore, be applied to reduce the load of sampling rate in a WLAN. Typically, in a WLAN, we have the following properties: 
- There is a total of $\mathrm{N}$ wireless nodes randomly located in a WLAN.

- We denote $\mathrm{K}$ as the number of random sparse wireless nodes.

- $\mathrm{K}$ is a random number and is much smaller than $\mathrm{N}$.

- We denote $[\mathrm{D}]_{\mathrm{N} \times 1}$ as the event vector.

- Each component of $[\mathrm{D}]_{\mathrm{N} \times 1}$ has a random value.

- Obviously $[D]_{N \times 1}$ is a sparse vector since $K \leq N$

- There are $M$ active monitoring wireless nodes trying to capture $K$ events.

- The number of events $\mathrm{K}$, the number of active wireless nodes $M$, and total of sources $\mathrm{N}$, have the following relation [32]:

$$
\mathrm{K} \leq \mathrm{M}<<\mathrm{N}
$$

Based on the proposed algorithm:

- We are able to organize a network traffic framework to help in ensuring the quality of service in a given wireless local area network.

- We can classify network traffic to allow us to organize traffic of packets into the traffic of packet classes or categories, on the basis of whether the traffic of packets matches specific criteria.

- We can classify network traffic as the foundation for enabling many QoS features in the WLANs.

- We can classify network traffic to a set of group traffic classes in order to apply specific QoS treatments. The QoS treatments might include faster forwarding packets from wireless nodes by intermediate wireless routers and switches, or reduced probability of the traffic being dropped due to lack of buffering resources.

- We can identify and categorize network traffic into traffic classes for different types of networks, effectively separating network traffic into different paths.

- We can classify network traffic by using class maps and policy maps with the Modular Quality of Service Command-Line Interface (MQC) method, in order to improve the level of QoS.

The RTNTM method works based on the following steps:

- $\quad$ Consider the pairs of measured QoS and the multiple attribute values as input for the CS.

- Estimates a multiple attribute compressed vector in which each element indicates the degree of QoS degradation caused by each wireless networking segment or device.

- Apply the estimated multiple attribute vector to identify the cause of QoS degradation.

The proposed algorithm is comprised based on the following steps:

- Apply segmentation algorithm to the WLAN in order to define the required number of segments [32]. The number of segments is calculated based on the following equation:

$$
\mathrm{M}=\mathrm{C} \log (5 \mathrm{~N}) \text { with } \mathrm{C} \text { between 20-25 }
$$

which $\mathrm{M}$ is the number of segments and $\mathrm{N}$ is the number of wireless nodes in the WLAN.

- Collect QoS vector for each segment.

- Generate compressed [QoS $]_{\mathrm{M} \times \mathrm{N}}$ matrix.

- Check the performance of the compressed [QoS $]_{\mathrm{M} \times \mathrm{N}}$ matrix based on the following condition:

$$
(1-\mathrm{C})\left\|[\mathrm{QoS}]_{\mathrm{M} \times \mathrm{N}} \mathrm{Y}\right\|_{2} \leq(1+\mathrm{C})\|\mathrm{Y}\|
$$

where $\mathrm{Y}$ is a random vector of $[\mathrm{QoS}]_{\mathrm{M} \times \mathrm{N}}$. If $[\mathrm{QoS}]_{\mathrm{M} \times \mathrm{N}}$ matrix satisfies the condition (2), we can go the next step. Otherwise, we need to improve the quality of $[\mathrm{QoS}]_{\mathrm{M} \times \mathrm{N}}$ matrix.

- Calculate the degradation degree of $[\mathrm{Q} o S]_{\mathrm{M} \times \mathrm{N}}$ matrix. 
- Calculate Packet Loss Matrix (PLS) and Mean Opinion Score (MOS). PLS is a quantitative measure of information lost over a WLAN and MOS is a good measure of QoS.

- Check the performance of the network based on MOS number. Table 1 compares the different quality levels of a WLAN to the different number of MOS.

Table 1. Different quality levels of a wireless local area network (WLAN) to the different number of Mean Opinion Score (MOS).

\begin{tabular}{cc}
\hline Qulity of Service (QoS) of a WLAN & Value of MOS \\
\hline Very Satisfied & $3.8-4$ \\
Satisfied & $3.5-3.7$ \\
Some segments are satisfied & $3.2-3.4$ \\
Many segments are dissatisfied & $2.9-3.1$ \\
Bad quality & Less than 2.9 \\
\hline
\end{tabular}

The proposed algorithm is based on the following sub-steps:

(1) Estimate the end-to-end QoS in the normal state.

(2) Calculate the degree of end-to-end QoS degradation by subtracting the estimated QoS in the normal.

(3) Subtract a baseline from then apply the CS to the data after subtracting it.

(4) Construct an estimation of QoS.

(5) Estimate the coefficients for CS with the least squares approach.

(6) Estimate QoS degradation vector.

(7) Identifies which attributes are causing the QoS degradation.

(8) Calculate degradation indicator.

(9) Calculate the correlation between latency and signal strength indicator (SSI).

The new RTNTM method has the following advantages:

- Detect, diagnose, and resolve network performance issues and errors either for each user or each networking device.

- Track response time, transmission rate, power management, availability and uptime of routers, switches and other networking devices.

- Analyze and monitor network bandwidth performance, modulation, protocol, network traffic, and traffic patterns.

- Manage and control bandwidth of each wireless user and application.

- Graphically display routing metrics, network performance metrics in real-time, based on dynamic routing protocol.

Figure 1 shows the proposed flowchart for the RTNTM algorithm.

The proposed RTNTM architecture allows reducing sampling rate, as well as network traffic. In addition, the proposed algorithm outperforms existing algorithms by improving QoS in WLANs. The proposed algorithm is established on the following steps:

- The input data set of a WLAN partition into a random number of clusters

- Iterate over all data vectors to determine clusters based on nearest subspace

- Compute the data vector's contribution to the total residual by re-assignment of the input data vectors to their found clusters

- The algorithm is repeated by alternate applications of Steps 2 and 3 until convergence.

In addition, the new RNTC method has the following advantages: 
- Analyze and monitor network bandwidth performance, modulation, protocol, network traffic, and traffic patterns.

- Manage and control bandwidth of each user, class, wireless node, and application.

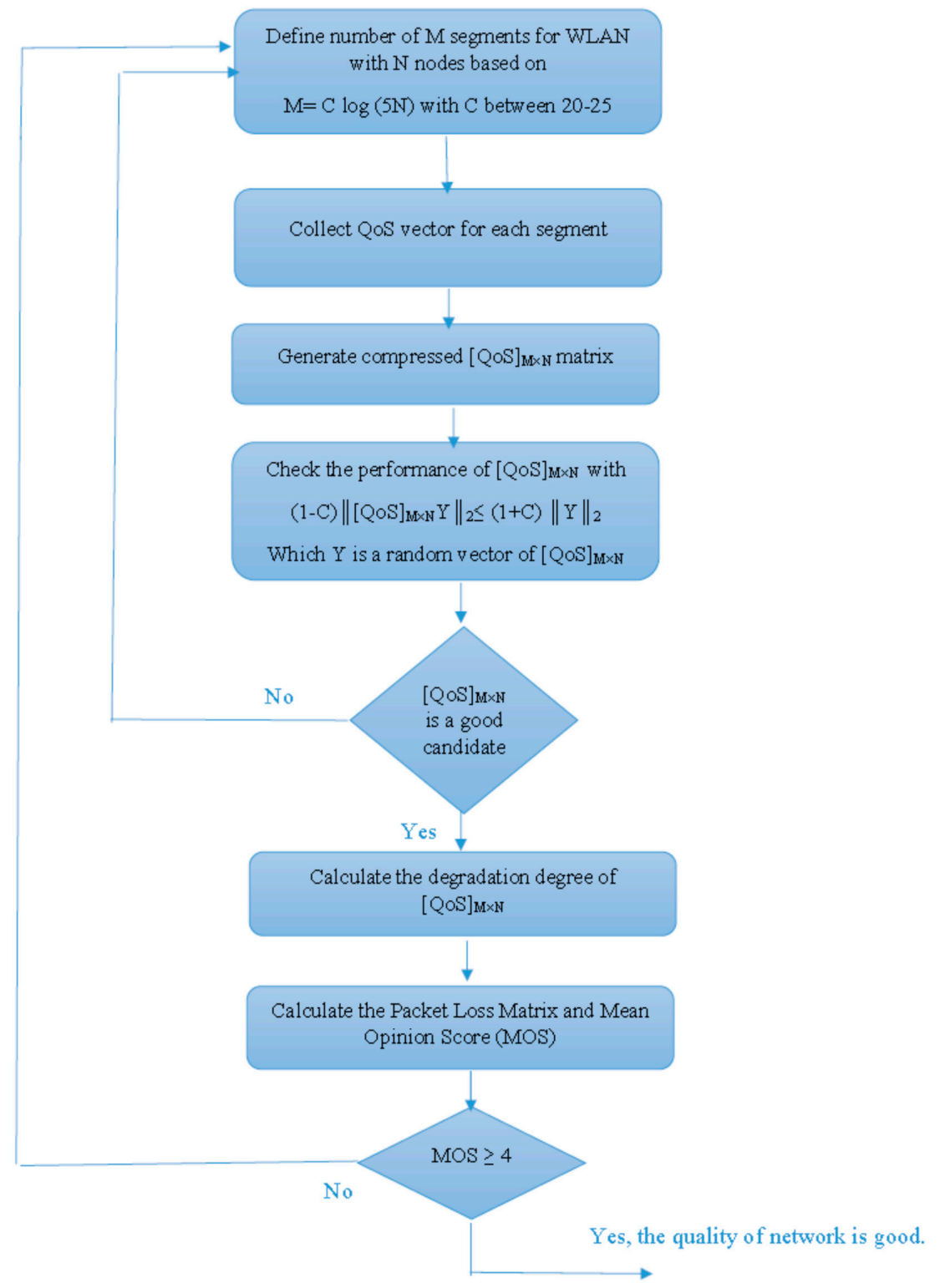

Figure 1. The proposed RTNTM algorithm.

\subsection{RTNTC Algorithm}

The proposed clustering techniques can be applied to packets from different wireless nodes that are quantitative, qualitative, or a mixture of both. In this algorithm, a cluster is a collection of packet objects that are similar to one another within the same cluster and dissimilar to the objects in other clusters. The proposed clustering algorithm can also be applied to large datasets of different wireless communication systems. The grouping is done by minimizing the sum of squares of distances between wireless nodes and the corresponding cluster centroid [32]. The proposed algorithm has the following advantages: (1) perform online clustering and classification; (2) establish strong background to expend the current WLANs to ultra-low-power WLANs. The proposed algorithm is defined based on the following assumptions: (1) total of $\mathrm{N}$ wireless nodes; (2) denote $\mathrm{K}$ as the number of random sparse 
wireless nodes; (3) $\mathrm{K}$ is a random number and is much smaller than $\mathrm{N}$; (4) denote $[\mathrm{D}]_{\mathrm{N} \times 1}$ as the packet vector in the WLAN; (5) assign each component of $[\mathrm{D}]_{\mathrm{N} \times 1}$ like a random value; (6) $[\mathrm{D}]_{\mathrm{N} \times 1}$ is a sparse vector since $\mathrm{K} \leq \mathrm{M}<<\mathrm{N}$; (7) there are $M$ active monitoring wireless nodes trying to capture $\mathrm{K}$ events.

Any WLAN signal D can be expressed as [32]:

$$
\mathrm{D}=\sum_{\mathrm{i}=1}^{\mathrm{N}} \mathrm{S}_{\mathrm{i}} \Psi_{\mathrm{i}}
$$

where $\mathrm{S}$ is the coefficient vector for $\mathrm{D}$ under the basis $\Psi=\left[\Psi_{1}, \Psi_{2}, \ldots, \Psi_{N}\right]$. If $\mathrm{D}$ has the most compact representation in $\Psi$, then $D$ should be compressed if acquired on the proper basis. The CS theory also proposes that rather than acquire the entire signal and then compress, it should be possible to capture the signal's useful information. Then, we can determine the compressed WLAN's signal W from the random matrix $\Phi$ based on the following equation [32]:

$$
[\mathrm{W}]_{\mathrm{M} \times 1}=[\Phi]_{\mathrm{M} \times \mathrm{N}}[\mathrm{D}]_{\mathrm{N} \times 1}
$$

The matrix $[\Phi]$ must satisfy the Restricted Isometry Property (RIP) with the following conditions [32]:

$$
1-\varepsilon \leq\|\Phi \alpha\|_{2} \leq 1+\varepsilon ;
$$

Then, we apply the following steps: (1) Apply segmentation algorithm to the WLAN in order to define the required number of classes. The number of classes is calculated based on the following equaltion [32]:

$$
\mathrm{M} \leq \frac{\mathrm{K}}{\mathrm{C}(\log N)}, \quad \text { with } \mathrm{C} \text { between 3-18 }
$$

which $\mathrm{M}$ is the number of classes and $\mathrm{N}$ is the number of wireless nodes in the WLAN.

(2) Collect QoS vector for each class; (3) Generate compressed [QoS $]_{\mathrm{M} \times \mathrm{N}}$ matrix; (4) Check the performance of the compressed [QoS $]_{\mathrm{M} \times \mathrm{N}}$ matrix based on the following condition [33]:

$$
(1-\mathrm{C})\left\|[\mathrm{QoS}]_{\mathrm{M} \times \mathrm{N}} \mathrm{Y}\right\|_{2} \leq(1+\mathrm{C})\|\mathrm{Y}\|_{2}
$$

where $\mathrm{Y}$ is a random vector of $[\mathrm{QoS}]_{\mathrm{M} \times \mathrm{N}}$. If $[\mathrm{QoS}]_{\mathrm{M} \times \mathrm{N}}$ matrix satisfies the condition (5) we can go the next step. Otherwise, we need to improve the quality of [QoS $]_{\mathrm{M} \times \mathrm{N}}$ matrix; (5) Calculate the Classification Matrix (CM), and identify the number of classes. This matrix sorts all cases from the wireless nodes into different categories, by determining whether the predicted value $(\mathrm{M})$ matched the actual value. All the cases in each category are then counted, and the totals are displayed in the CM matrix. The classification matrix in WLANs is a standard tool for evaluation of statistical models and is sometimes referred to as a confusion matrix of a WLAN. In addition, the CA is an important tool for assessing the performance of the WLANs. The propped algorithm is based on the performance of the $\mathrm{CM}$ for the following steps:

- The CM sorts all cases from the packets into classes, by determining whether the predicted value of each packet matched the actual value of each packet.

- All the packets in each class are then counted, and the totals are displayed in the CM.

- The classification matrix is applied to evaluate the performance of the classification algorithm.

The grouping is done by minimizing the sum of squares of distances between data and the corresponding cluster centroid. Figure 2 shows the proposed flowchart for the RTNTC algorithm. 


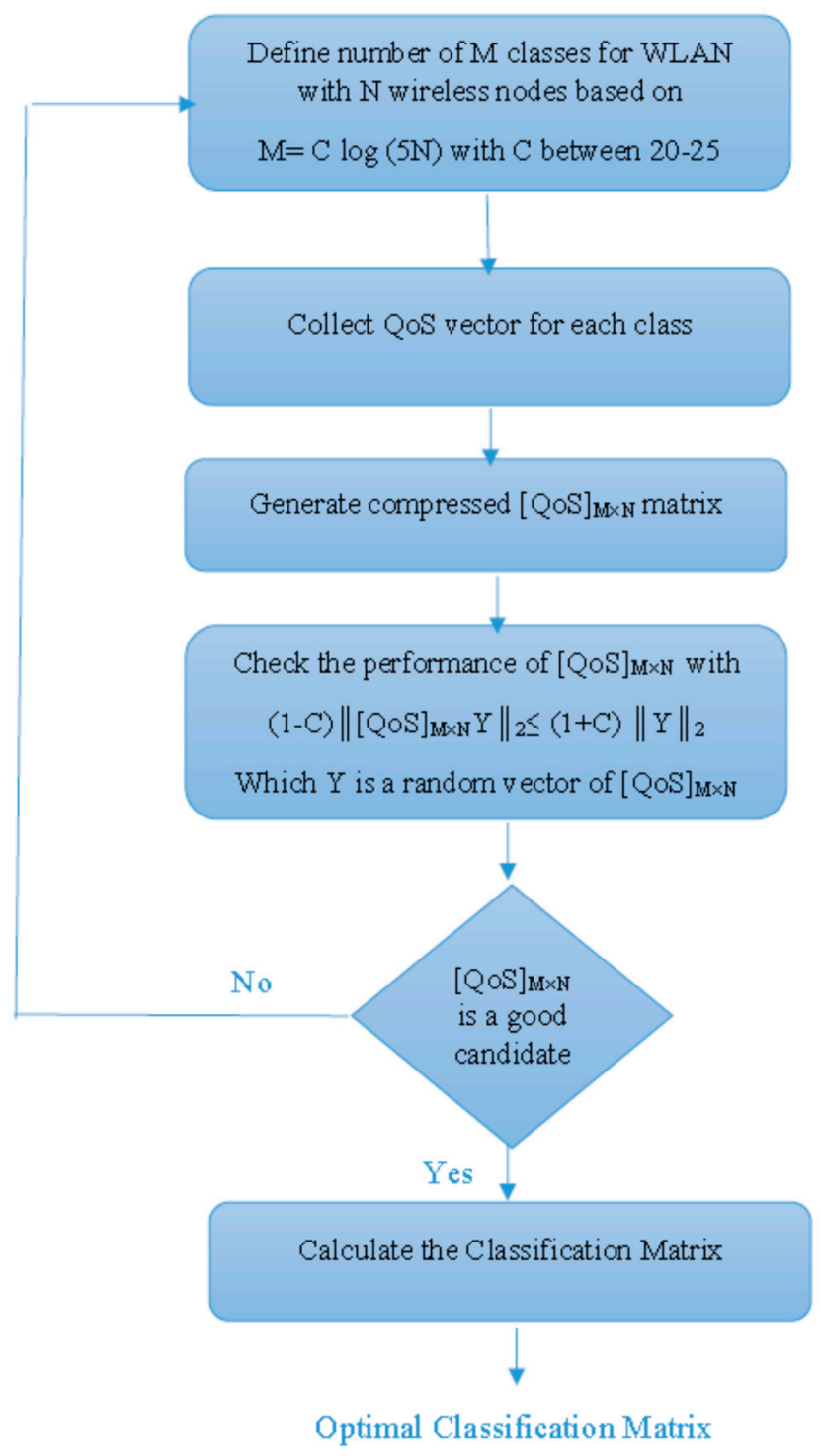

Figure 2. The proposed Real-Time Network Traffic Classification (RTNTC) algorithm.

We validate the proposed algorithm's performance with the following techniques:

- $\quad$ Principal Component Analysis (PCA) [33].

- K-Nearest Neighbors (K-NN) [33].

- K-Means clustering algorithm.

Then, dimensionality reduction methods followed by sorting the data using the K-NN and K-Means clustering algorithms are evaluated for the performance of the proposed algorithm.

The proposed algorithm is established based on the following steps:

- The proposed RTNTC classification algorithm uses a set of features or parameters of packets to characterize each class, where these features should be relevant to the suitable classes. The set of known objects is called the training set, because it is used by the proposed classification algorithm to learn how to classify different packets.

- The proposed RTNTC algorithm is based on two phases to construct a robust classifier. In the training phase, the training set is used to decide how the parameters ought to be weighted and combined, in order to separate the various classes of packets. In the application phase, 
the weights determined in the training set are applied to a set of packet objects that do not have known classes in order to determine what their classes are likely to be in any wireless path or wireless device.

- The proposed RTNTC algorithm is based on a nearest-neighbor approach. In this method, one packet simply finds in the $\mathrm{N}$-dimensional feature space the closest packet from the training set to an object being classified.

The proposed algorithm has the following advantages: (1) Perform online clustering and classification; (2) Establish strong background to expend the current WLANs to ultra-low-power WLANs; (3) Classify network traffic to allow us to organize traffic of packets into traffic of packets classes or categories on the basis of whether the traffic of packets matches specific criteria; (4) Classify network traffic is the foundation for enabling many QoS features on the WLANs; (5) Classify network traffic to a set of group traffic classes in order to specific QoS treatments; (6) Identify and categorize network traffic into traffic classes for different types of networks, effectively separating network traffic into different paths; (7) Classify network traffic by using class maps and policy maps with the Modular Quality of Service Command-Line Interface (MQC) method in order to improve the level of QoS; (8) The proposed algorithm is easy to implement; (9) The proposed algorithm can also give quite good results if the features are chosen carefully; (10) The proposed algorithm is weighted carefully in the computation of the distance between wireless nodes and wireless devices

\section{Simulation Results}

In the simulation methodology, the following conditions are used: (1) a set of features or parameters of packets to characterize each class, where these features should be relevant to the suitable classes; (2) The set of known objects for the training dictionary to learn how to classify different packets; (3) In the training phase, the training set is used to decide how the parameters ought to be weighted and combined in order to separate the various classes of packets; (4) In the application phase, the weights determined in the training set are applied to a set of packet objects that do not have known classes in order to determine what their classes are likely to be in any wireless path or wireless device. To evaluate the performance of our methods, the following dataset has been used:

- Dataset was obtained through the following experiments in three WLANs.

- The experiment participants used various types of wireless networking devices in three WLANs, each was capable of measuring QoS and accessing internet sites from various locations.

- $\quad$ For each access, the latency of WLAN was measured and collected as the end-to-end QoS.

- At the same time, the attribute information of the WLAN was also collected.

- The QoS of WLANs was measured using the speed test application. The proposed architecture allows reducing DDP to $15 \%$, BER to $14 \%$ at each wireless node, FDR to $25 \%$, and PD to $15 \%$, which are good records for WLANs. The proposed architecture increased DT to $22 \%$ and S/N ratio to $17 \%$, and $10 \%$ accuracy of wireless transmission.

Figure 3 shows data (packet) delay probability versus maximum packet size. It can be observed from Figure 2 that the proposed algorithms exhibit excellent performance in data (packet) delay probability (in our work to $15 \%$ ). Figure 4 plots the data throughput versus of the maximum packet size. It can be noted that the good level data throughput is obtained by increasing the of the maximum packet size (in our work to $22 \%$ ).

Figure 5 shows the $\mathrm{S} / \mathrm{N}$ versus the number of users. It can be seen from Figure 4 that $\mathrm{S} / \mathrm{N}$ ratio can be increased to $17 \%$ without sacrificing of the performance in the WLAN. Figure 5 plots the normalized bit error rate versus number of packets. It can be seen from Figure 6 that bit error rate can be minimized to $15 \%$ in our work. Figure 7 shows accuracy versus of the number of clusters. It can be observed from Figure 2 that the proposed algorithms exhibit excellent performance in accuracy (in our work to $10 \%$ ). Figure 8 plots the FDR versus of the number of clusters. It can be noted that the 
good level data FDR is obtained by increasing the of the maximum packet size (in our work to $25 \%$ ). Figure 9 shows the probability versus of packet delay. It can be seen from Figure 4 that packet delay can be decreased to $15 \%$ without sacrificing of the performance in the WLAN.

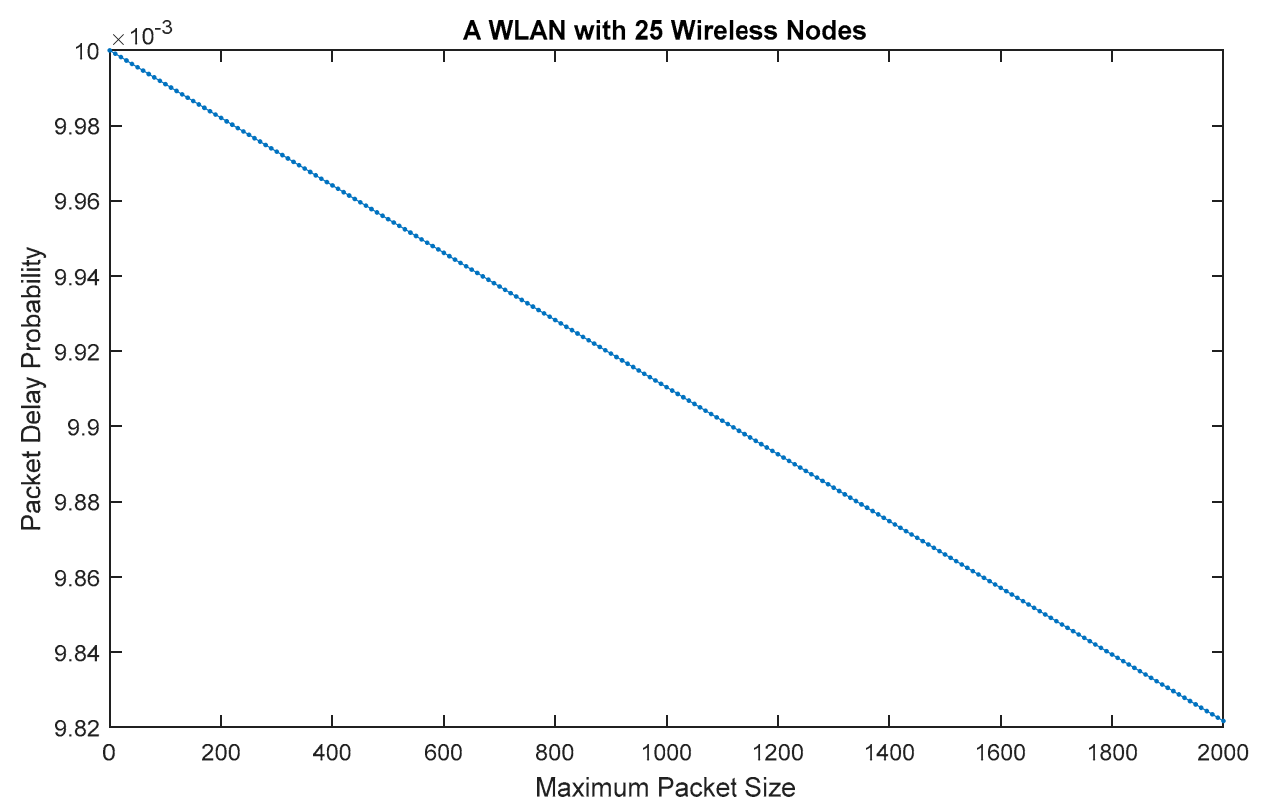

Figure 3. Data (packet) delay probability versus maximum packet size.

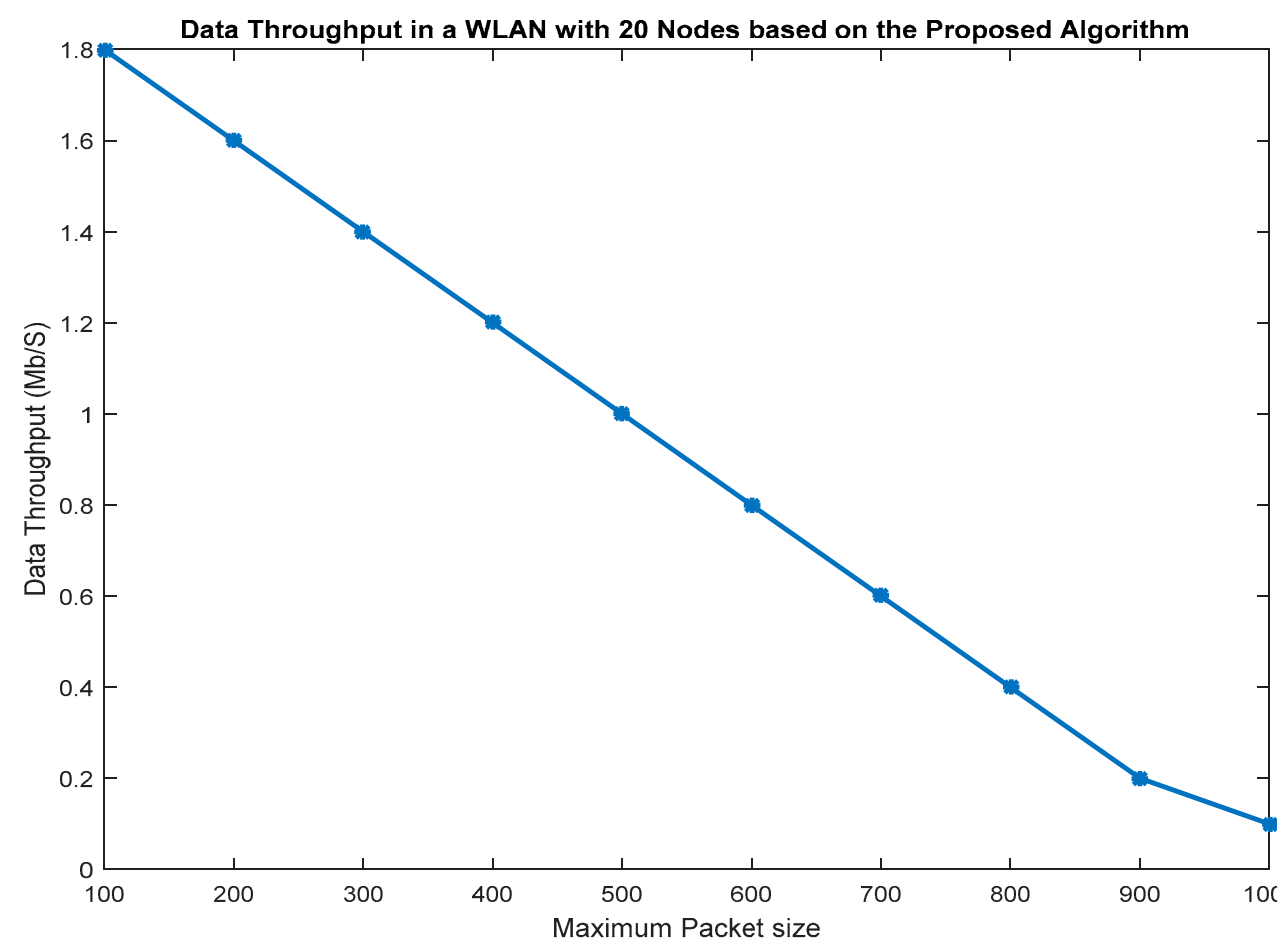

Figure 4. Data throughput versus of the maximum packet size. 


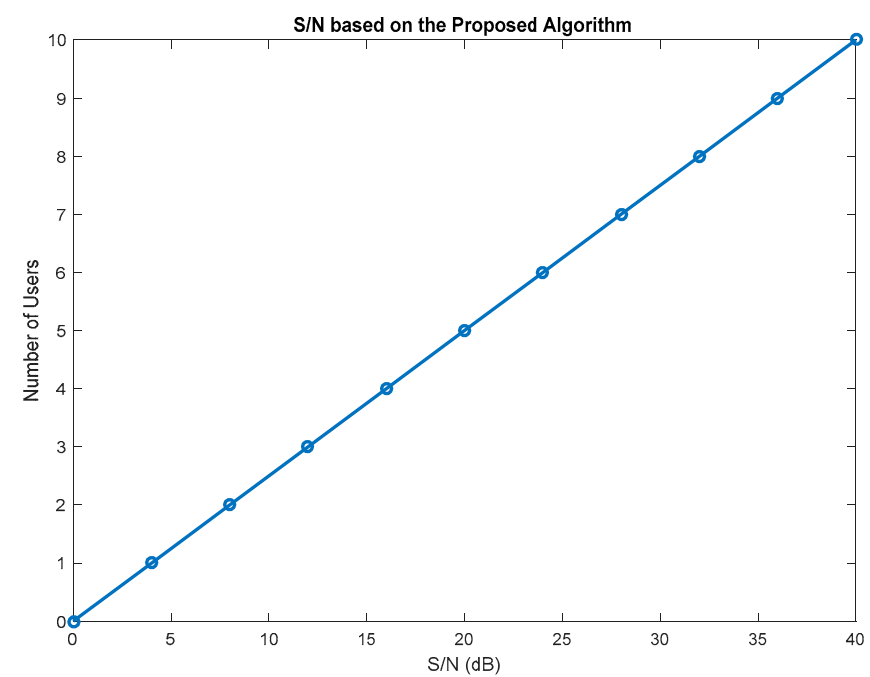

Figure 5. Signal to Noise (S/N) vs the number of users.

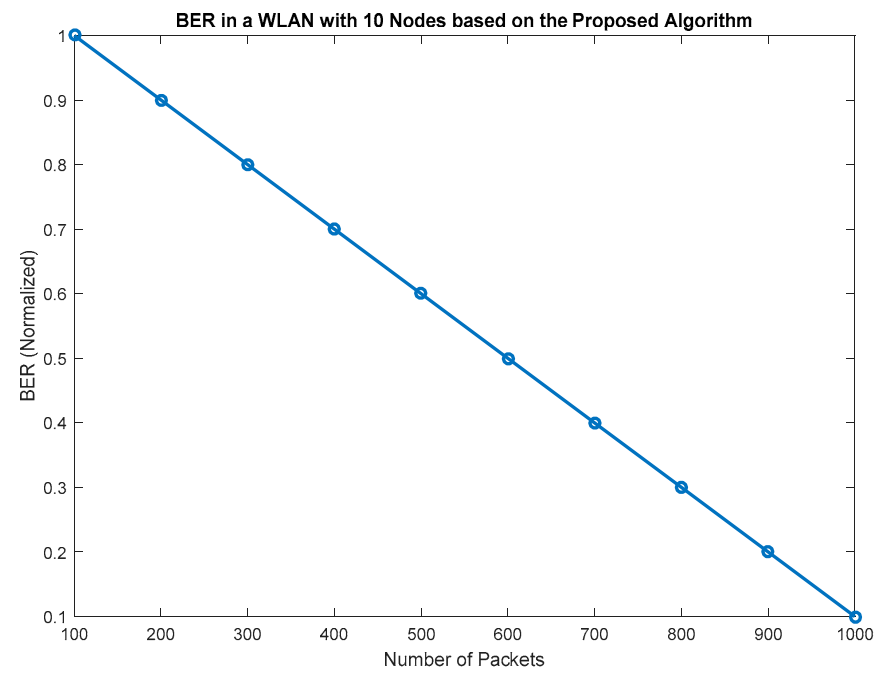

Figure 6. Normalized bite error rate vs number of packets.

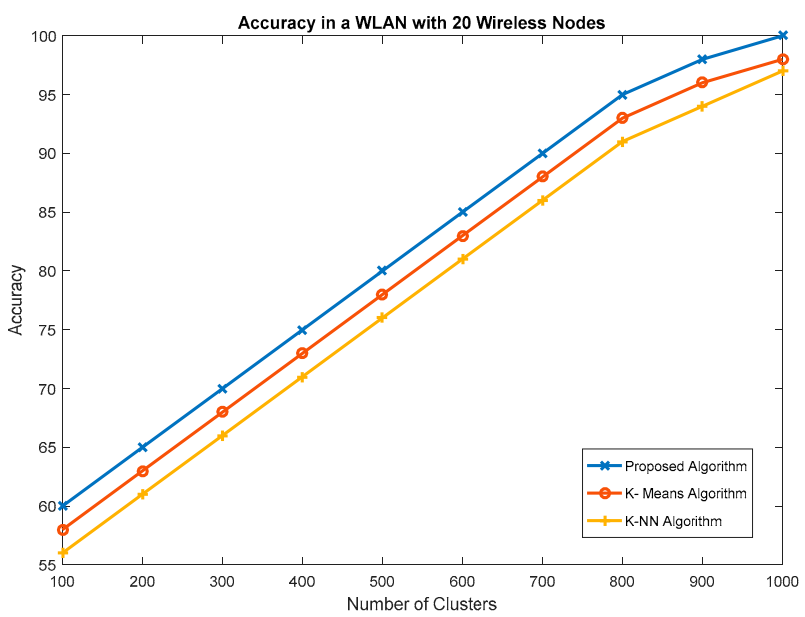

Figure 7. Accuracy versus of the number of clusters. 


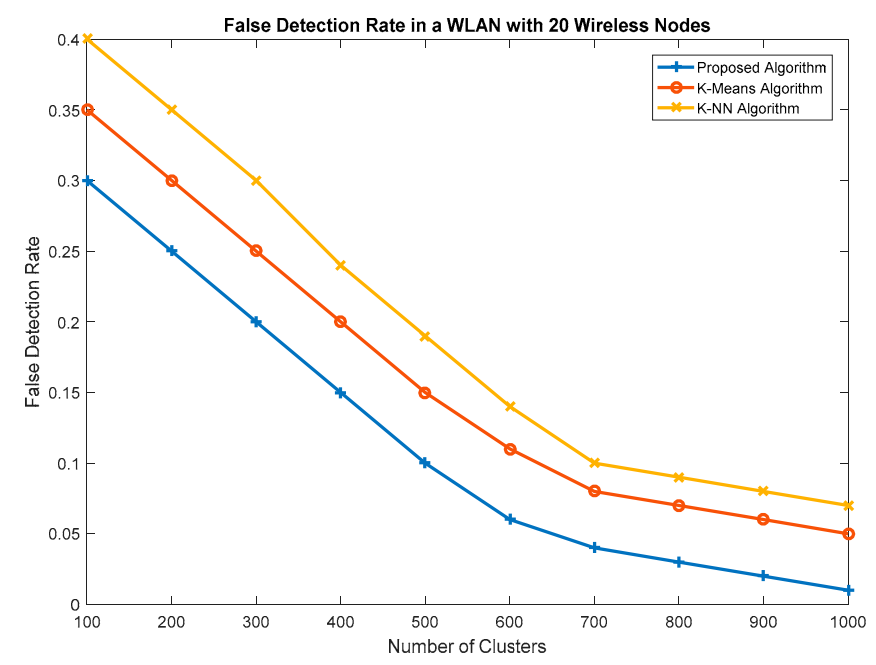

Figure 8. False Detection Rate (FDR) versus of the number of clusters.

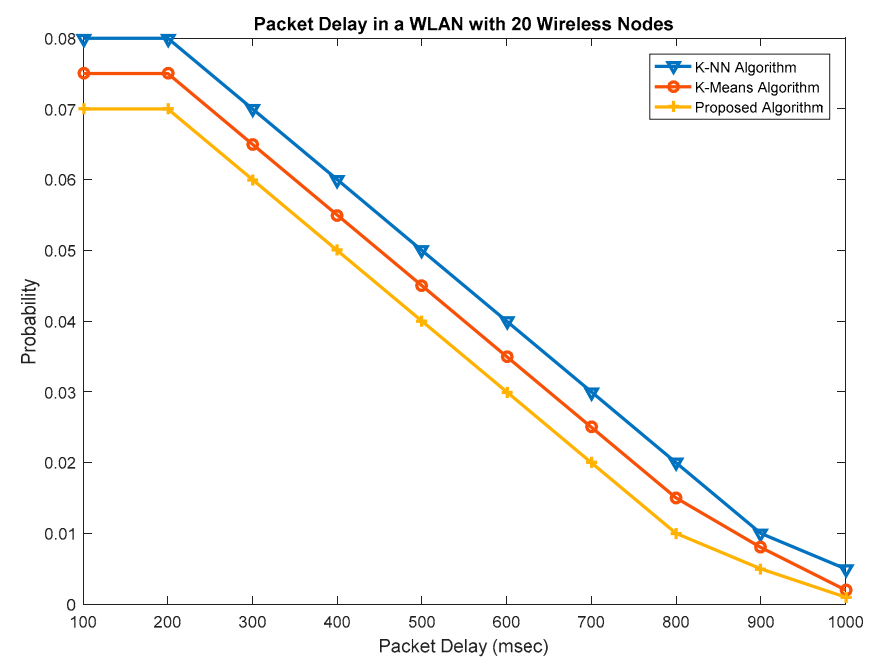

Figure 9. Probability versus of packet delay.

\section{Conclusions}

Wireless local area networks are important types of wireless networks, such as wireless personal area networks, wireless metropolitan networks, and wide area networks. These networks suffer from important problems, such as network load balancing, large amount of energy, and load of sampling. Network traffic classification is an important tool that is required to enable any traffic management operations, from differentiating traffic pricing and treatment to security operations. The information provided by traffic classification in a WLAN is extremely important for improving the quality of the wireless network. The existing network traffic classification techniques suffer from important problems such as network load balancing, large amount of energy, and load of sampling. This paper presented a new networking traffic approach based on CS which involves a random sampling approach instead of SNS theory. In addition, this paper presented a new network traffic classification approach based on CS which involves a random sampling approach instead of SNS theory. The proposed architecture allowed reducing of Data Delay Probability (DDP) to 15\%, Bit Error Rate (BER) to 14\% at each wireless node, False Detection Rate (FDR) to 25\%, and Packet Delay (PD) to 15\%, which are good records for WLANs. The proposed architecture increased Data Throughput (DT) to $22 \%$ and Signal to Noise (S/N) ratio to $17 \%$, and $10 \%$ accuracy of wireless transmission. The proposed architecture provides a good background for establishing high qualified wireless local area networks. In addition, the proposed 
architecture enabled continuous data acquisition and compression of WLAN's signals that are suitable for a variety of other wireless networking applications to solve difficult wireless network management problems. The proposed algorithm outperformed the existing algorithms by achieving a good level of QoS and BER at each wireless node.

Conflicts of Interest: The authors declare no conflict of interest.

\section{References}

1. Chanthong, R.; Wuttidittachotti, P.; Daengsi, T. A study of G.711 and ILBC over WLAN 802.11n with EDCA. In Proceedings of the 2015 International Conference on Information Networking (ICOIN), Siem Reap, Cambodia, 12-14 January 2015; pp. 189-193.

2. Hunger, A.; Klein, P.A. Equalizing latency peaks using a redundant multipath-TCP scheme. In Proceedings of the 2016 International Conference on Information Networking (ICOIN), Kota Kinabalu, Malaysia, 13-15 January 2016; pp. 184-189.

3. Li, B.; Kim, K. A real-time routing protocol for (mk)-firm streams in wireless sensor networks. In Proceedings of the 2013 IEEE Eighth International Conference on Intelligent Sensors Sensor Networks and Information Processing, Melbourne, Australia, 2-5 April 2013; pp. 129-134.

4. Salah, H.B.; Benzina, A.; Khalgui, M. Verification of Reconfigurable NoC under Quality of Service Constraints. In Proceedings of the 2016 IEEE 40th Annual Computer Software and Applications Conference (COMPSAC), Atlanta, GA, USA, 10-14 June 2016; Volume 1, pp. 329-334.

5. Lee, H.; Park, K.J.; Ko, Y.B.; Choi, C.H. Wireless LAN with medical-grade QoS for e-healthcare. J. Commun. Netw. 2011, 13, 149-159. [CrossRef]

6. Son, S.; Park, K.-J.; Park, E.-C. Admission control in medical-grade WLAN. In Proceedings of the 2014 Sixth International Conference on Ubiquitous and Future Networks (ICUFN), Shanghai, China, 8-11 July 2014; pp. 541-542.

7. Zen, H.; Habibi, D.; Ahmad, I. Self-restraint Admission Control for adhoc WLANs. In Proceedings of the ATNAC 2008 Telecommunication Networks and Applications Conference, Adelaide, Australia, 7-10 December 2008; pp. 186-191. [CrossRef]

8. Liu, Y.; Pawar, S.S.; Assi, C.; Agarwal, A. Dynamic Admission and Congestion Control for Real-time Traffic in IEEE 802.11e Wireless LANs. In Proceedings of the 2006 IEEE International Conference on Wireless and Mobile Computing, Networking and Communications, Montreal, QC, Canada, 19-21 June 2006; pp. 419-426. [CrossRef]

9. Mamaghanian, H.; Khaled, N.; Atienza, D.; Vandergheynst, P. Compressed Sensing for Real-Time EnergyEfficient ECG Compression on Wireless Body Sensor Nodes. IEEE Trans. Biomed. Eng. 2011, 58, 2456-2466. [CrossRef] [PubMed]

10. Chen, F.; Chandrakasan, A.P.; Stojanovic, V.M. Design and Analysis of a Hardware-Efficient Compressed Sensing Architecture for Data Compression in Wireless Sensors. IEEE J. Solid-State Circuits 2012, 47, 744-756. [CrossRef]

11. Chen, F.; Chandrakasan, A.P.; Stojanovic, V. A signal-agnostic compressed sensing acquisition system for wireless and implantable sensors. In Proceedings of the 2010 IEEE Custom Integrated Circuits Conference (CICC), San Jose, CA, USA, 19-22 September 2010; pp. 1-4.

12. Balouchestani, M.; Raahemifar, K.; Krishnan, S. Compressed Sensing in Wireless Sensor Networks: Survey. Can. J. Multimed. Wirel. Netw. 2011, 2, 1-4.

13. Balouchestani, M.; Raahemifar, K.; Krishnan, S. Power Management of Wireless Sensor Networks with Compressed Sensing Theory. In Proceedings of the 16th IEEE International Conference on Networks and Optical Communications and 6th Conference on Optical Cabling and Infrastructure (OC\&I) Northumbria University, Newcastle upon Tyne, UK, 9-10 July 2011; pp. 122-125.

14. Balouchestani, M.; Raahemifar, K.; Krishnan, S. Concepts for Designing Low-Power Wireless Sensor Networks with Compressed Sensing Theory. In Proceedings of the International Conference on Communication and Broadband Networking (ICCBN 2011), Kuala Lumpur, Malaysia, 3-5 November 2011; pp. 47-51.

15. Ye, H.; Walsh, G.C.; Bushnell, L.G. Real-time mixed-traffic wireless networks. IEEE Trans. Ind. Electron. 2001, 48, 883-890. [CrossRef] 
16. Yeon, H.J.; Sahu, S.K.; Park, W.; Lee, J.W.; Lee, J. Adaptive transmission scheme using dynamic aggregation and fragmentation in WLAN MAC. In Proceedings of the 2014 IEEE Wireless Communications and Networking Conference (WCNC), Istanbul, Turkey, 6-9 April 2014; pp. 1532-1537. [CrossRef]

17. Podolanko, J.; Datta, S.; Das, S.K. Performance analysis of real-time traffic over 802.11n Wireless Local Area Networks: An experimental study. In Proceedings of the 2014 International Wireless Communications and Mobile Computing Conference (IWCMC), Nicosia, Cyprus, 4-8 August 2014; pp. 453-457. [CrossRef]

18. Frantti, T.; Majanen, M. Real-time traffic control for multihomed devices. In Proceedings of the International Conference on Information Networking 2013 (ICOIN), Bangkok, Thailand, 28-30 January 2013; pp. 113-118. [CrossRef]

19. Kanoun, K.; Mamaghanian, H.; Khaled, N.; Atienza, D. A real-time compressed sensing-based personal electrocardiogram monitoring system. In Proceedings of the Design, Automation \& Test in Europe Conference \& Exhibition (DATE), Grenoble, France, 14-18 March 2011; pp. 1-6.

20. Haupt, J.; Bajwa, W.U.; Rabbat, M.; Nowak, R. Compressed Sensing for Networked Data. IEEE Signal Process. Mag. 2008, 25, 92-101. [CrossRef]

21. Luo, J.; Xiang, L.; Rosenberg, C. Does compressed sensing improve the throughput of wireless sensor networks? In Proceedings of the IEEE International Conference Communications (ICC), Cape Town, South Africa, 23-27 May 2010; pp. 1-6.

22. Yang, X.; Tao, X.; Dutkiewicz, E.; Huang, X.; Guo, Y.J.; Cui, Q. Energy-Efficient Distributed Data Storage for Wireless Sensor Networks Based on Compressed Sensing and Network Coding. IEEE Trans. Wirel. Commun. 2013, 12, 5087-5099. [CrossRef]

23. Balouchestani, M.; Raahemifar, K.; Krishnan, S. Robust Wireless Sensors with Compressed Sensing Theory. Commun. Comput. Inf. Sci. J. Springer 2012, 293, 608-619.

24. Ermis, E.B.; Saligrama, V. Dynamic thresholding for distributed multiple hypotheses testing. In Proceedings of the SSP '07. IEEE/SP 14th Workshop Statistical Signal Processing, Madison, WI, USA, $26-29$ August 2007; pp. 675-679.

25. Al-Karaki, J.N.; Kamal, A.E. Routing techniques in wireless sensor networks: A survey. IEEE Wirel. Commun. 2004, 11, 6-28. [CrossRef]

26. D'Andrea, E.; Ducange, P.; Lazzerini, B.; Marcelloni, F. Real-Time Detection of Traffic from Twitter Stream Analysis. IEEE Trans. Intell. Transp. Syst. 2015, 16, 2269-2283. [CrossRef]

27. Wei, H.; Sun, B.; Jing, M. BalancedBoost: A hybrid approach for real-time network traffic classification. In Proceedings of the 2014 23rd International Conference on Computer Communication and Networks (ICCCN), Shanghai, China, 4-7 August 2014; pp. 1-6. [CrossRef]

28. Tongaonkar, A.; Keralapura, R.; Nucci, A. SANTaClass: A Self Adaptive Network Traffic Classification system. In Proceedings of the IFIP Networking Conference, Brooklyn, NY, USA, 22-24 May 2013; pp. 1-9.

29. Choudhury, P.; Kumar, K.R.P.; Athithan, G.; Nandi, S. Analysis of VBR coded VoIP for traffic classification. In Proceedings of the 2013 International Conference on Advances in Computing, Communications and Informatics (ICACCI), Mysore, India, 22-25 August 2013; pp. 90-95. [CrossRef]

30. Balouchestani, M.; Raahemifar, K.; Krishnan, S. New sampling approach for wireless ECG systems with compressed sensing theory. In Proceedings of the IEEE International Symposium Medical Measurements and Applications Proceedings (MeMeA), Gatineau, QC, Canada, 4-5 May 2013; pp. 213-218.

31. Balouchestani, M.; Raahemifar, K.; Krishnan, S. Low power wireless body area networks with compressed sensing theory. In Proceedings of the IEEE 55th International Midwest Symposium on Circuits and Systems (MWSCAS), Boise, ID, USA, 5-8 August 2012; pp. 916-919.

32. Balouchestani, M.; Raahemifar, K.; Krishnan, S. New Testing Method in Wireless Sensor Networks with Compressed Sensing Theory. In International Conference on Computer Communication and Management (ICCCM2011); IACSIT Press: Sydney, Australia, 2011; Volume 5, pp. 1-6.

33. Balouchestani, M.; Krishnan, S. Advanced K-means clustering algorithm for large ECG data sets based on a collaboration of compressed sensing theory and K-SVD approach. J. Signal Image Video Process. 2016, 10, 113-120. [CrossRef]

(C) 2018 by the author. Licensee MDPI, Basel, Switzerland. This article is an open access article distributed under the terms and conditions of the Creative Commons Attribution (CC BY) license (http:/ / creativecommons.org/licenses/by/4.0/). 